\title{
Callyntra inconsiderata sp. nov. (Coleoptera: Tenebrionidae), una nueva especie para la costa de Chile Central
}

\section{Callyntra inconsiderata sp. nov. (Coleoptera: Tenebrionidae), a new species for the coast of Central Chile}

\author{
Álvaro ZúNIIGA-REINOSO ${ }^{1,2}$ \\ 'Laboratorio de Genética y Evolución, Departamento de Ciencias Ecológicas, Facultad de Ciencias, Universidad de Chile; \\ alzure@gmail.com \\ ${ }^{2}$ Red Chilena Entomológica de la Conservación, RECEC
}

\begin{abstract}
RESUMEN
Callyntra Solier, 1836, es un género endémico de Chile y Argentina que contiene 21 especies descritas hasta la fecha. Mediante recolecciones y revisión de material proveniente de la costa de las Regiones V y VI, se encontró con especímenes asignados a C. laticollis, pero que morfológicamente diferían de la especie asignada y del resto de las especies del género. Por lo tanto, en este artículo se describe $C$. inconsiderata sp. nov y se discute sobre su relación con otras especies de Callyntra, su variabilidad morfológica, dimorfismo sexual y conservación.
\end{abstract}

Palabras clave: distribución, Callyntra laticollis, Callyntra paulseni, Nycteliini, Pimeliinae, conservación.

\begin{abstract}
Callyntra Solier, 1836, an endemic genus of Chile and Argentina,contains 21 valid species. Through field sampling and review of material from the coast of the Regions V and VI, we find specimens assigned to C. laticollis morphologically differentiated from the assigned species. Also, their morphology differ from all other nominal species of the genus. Therefore, in this article we describe $C$. inconsiderata sp. nov. and discuss regarding the relationship with other species of Callyntra, morphological variability, sexual dimorphism and conservation.
\end{abstract}

KeYwords: distribution, Callyntra laticollis, Callyntra paulseni, Nycteliini, Pimeliinae, conservation.

\section{INTRODUCCIÓN}

El género Callyntra Solier, 1836incluye a un conjunto de especies endémicas de Chile (Flores \& Vidal, 2000a) y una especie compartida con Argentina (Roig-Juñent et al., 2004). Luego de una historia taxonómica inestable, el género fue establecido como grupo natural dentro de la tribu Nycteliini (Flores, 2000), lo que conllevo a una revisión y revalidación del mismo (Flores \& Vidal, 2000a) y se establecieron las relaciones filogenéticas entre las especies basado en morfología (Flores \& Vidal, 2000b). La distribución del género en Chile, abarca desde $32^{\circ}$ a los $39^{\circ}$ latitud sur, y las especies se pueden encontrar en ambas cordilleras y terrazas costeras (Flores \& Vidal, 2000a; 2000b). A pesar de ser un grupo taxonómicamente antiguo y bien estudiado (ver Flores \& Vidal, 2000a), recientemente se han descrito nuevas especies, como es el caso de $C$. hibrida (Zúñiga-Reinoso \& Cid-Arcos, 2013), C. zambranoi y $C$. rufocastanea (Aceituno \& Guerrero, 2014), C. pehuenche
(Zúñiga-Reinoso \& Cid-Arcos, 2015), teniendo en la actualidad 21 especies descritas (ver Flores \& Vidal, 2000 a y b; Zúñiga-Reinoso \& Cid-Arcos, 2013; Aceituno \& Guerrero, 2014). Recientemente, se capturaron y revisaron ejemplares provenientes de la costa de la región V y VI de Chile, que tradicionalmente eran asignadas a Callyntra laticollis Philippi \& Philippi, 1864 (Flores \& Vidal, 2000a), pero que morfológicamente difiere del material tipo de la especies. Por lo tanto, el objetivo de este trabajo es realizar una revisión exhaustiva de este material y proponerlo como una nueva especie no descrita para el género, en base a su morfología externa y de la estructura genital del macho.

\section{MATERIALES Y METODOS}

El material dispuesto para este estudio fue revisado bajo lupa estereoscópica y comparado con: 1) el Lectotipo de C. laticollis que se encuentra depositado en las colecciones 
entomológicas del Museo Nacional de Historia Natural, 2) Una serie de especímenes de $C$. paulseni, 3) con la descripción original de $C$. laticollis de Philippi \& Philippi (1864) y 4) las descripciones y fotografías entregadas por Flores \& Vidal (2000a) para C. laticollis y C. paulseni.

Por otro lado, una parte del material fue debidamente limpiado, montado en seco y etiquetado. Mientras que otra parte fue conservada en alcohol 99.8\%. Para la descripción de la morfología externa se siguió a Flores \& Vidal (2000a). Para establecer las diferencia entre las especies, según lo propuesto por Flores (1996), se examinó la genitalia de los machos, la que fue comparada con la de especies morfológicamente cercanas. Para esto los ejemplares machos fueron ablandados con agua para la extracción de la genitalia y posteriormente limpiadas con una solución de $\mathrm{KOH}$ al $5 \%$ por 5 minutos. El edeago fue suspendido en solución de Hood en tubos eppendorf de 0,2 ml. La longitud fue tomada en vista dorsal, desde la cabeza hasta el ápice de los élitros. Los dibujos fueron realizados y escalados utilizando el programa Corel Draw5x. Finalmente, los ejemplares examinados $\mathrm{y}$ asignados a la serie tipo se encuentran depositados en las siguientes colecciones:

MNHN - Museo Nacional de Historia Natural, Santiago, Chile.

MZUC- Museo de Zoología, Universidad de Concepción, Concepción, Chile.

IEUM- Colección Entomológica, Instituto de Entomología, Universidad Metropolitana de Ciencias de la Educación., Santiago, Chile.

GEVOL - Laboratorio de Genética y Evolución, Departamento de Ciencias Ecológicas, Facultad de Ciencias, Universidad de Chile, Santiago, Chile.

CPMC - Colección Particular de Mauricio Cid, Talca, Chile. CPPP - Colección Particular de Pablo Pinto, Valparaíso, Chile.

CPSL - Colección Particular de Sebastián Larrea, Valparaíso, Chile.

\section{RESULTADOS}

Familia Tenebrionidae Latreille, 1802

Subfamilia Pimelinae Latreille, 1802

Tribu Nycteliini Solier, 1834

Género Callyntra Solier, 1836

Callyntra inconsiderata Zúñiga-Reinoso sp. nov. (Fig. 1 y 2).

SinONimia.Callyntra laticollis en Flores \& Vidal (2000a): p. 1056, Figura 12 (pronoto) y en Zúñiga-Reinoso \& CidArcos (2014): p. 134, Figura 2b (genitalia vista lateral).

Holotipo: Chile: O'Higgins: Cardenal Caro: Navidad: Pupuya (3358'34'S-7151'32”O). 6. I. 2012. col. A. Zúñiga y D. Boric. $1 \delta^{\Uparrow}$ (Fig. 2). (MNHN Tipo $\left.N^{\circ} 7916\right)$.
Diagnosis. Los caracteres diagnósticos de $C$. inconsiderata (ver Fig. 1) son entregados conjuntamente con los caracteres que difieren de $C$. paulseni, debido a que es la especie morfológicamente mas cercana, siendo las características de C. paulseni entregadas en paréntesis: Proyección lateral del pronoto con punta romba (aguzada), cuerpo sin pilosidad, excepto en el declive elitral (cuerpo completamente cubierto de pilosidad), carinas pronotales levantada y gruesa (poco levantadas), patas dorsalmente glabras o pilosidad leve (completamente peludas), borde lateral del pronoto grueso (delgado), arrugas marcada entre carina y borde lateral del pronoto (arrugas leves), espacio intercarinal del pronoto con arrugas profundas y longitudinales en machos (leves y desordenadas).

Medidas del Holotipo. Largo máximo: $170 \mathrm{~mm}$, ancho máximo pronoto: $75 \mathrm{~mm}$, largo mínimo pronoto: $35 \mathrm{~mm}$, ancho máximo élitros: $65 \mathrm{~mm}$, largo máximo élitros: 110 $\mathrm{mm}$.

Paratipos: Chile: O'Higgins: Cardenal Caro: Navidad: Pupuya. 6.I. 2012. col. A. Zúñiga y D. Boric.1§/3 9 (MNHN Tipo $\mathrm{N}^{\circ}$ 7917-7920). 23.XI.1984. col. J.C. Ramírez. 1ठ (CPMC). El Maitén. 11.XI.2012. col. G. Aceituno. 4ð/ 3 우 (CPSL).30.XI.2013. col. P. Pinto. $1 \hat{\jmath} / 19$ (MNHN Tipo $\mathrm{N}^{\circ}$ 7921-7922), 1ð (GEVOL) . Matanzas. 14.XI.1976. col K. Lohnman. 1ð (IEUM) 15.XI.1976. col J. Simonetti. 2 ㅇ (IEUM). Navidad. 3.XI.1968. col. Moyano. 29 (MZUC Tipo $N^{\circ}$ 43277- 43278). col. Retamales. 3ふ/ 2 ㅇ (MZUC Tipo $\mathrm{N}^{\circ}$ 43279-43283). 19.XII.2004. col. C. Fortino 1 q (CPMC), $1 \hat{\jmath}$ (CPPP). Región de Valparaíso: Provincia de San Antonio: Santo Domingo, Bucalemu (Ruta G-80-1). 23.XI.2013. col. N. Peñaloza. 19 (MNHN Tipo $N^{\circ}$ 7923).

Otro Material Examinado.Chile: O'Higgins: Cardenal Caro: Navidad:Matanzas. 18. IX. 1981. col D. Rodríguez. $1 \delta$ [examinado en Flores \& Vidal, 2000a] (MNHN). El Maitén. XI.2012. col. C. Fortino. 1 (GEVOL). 30.XI.2013. col. P. Pinto. $1 ð$ (GEVOL).

Distribución. Chile: Regiones V y VI. Provincias de Cardenal Caro y San Antonio. Esto corresponde área de endemismo Desierto de Coquimbo Sur (Flores \& Vidal 2000b), que en esta latitud abarca las zonas costeras.

Etimología. El epíteto específico inconsiderata es un adjetivo singular femenino del latín que significa desconsiderada y hace referencia a que esta especie no fue considerada como especie diferente de $C$. laticollis en la revisión de Flores \& Vidal (2000a), a pesar de tener material de esta especie en la serie revisada.

DesCRIPCIÓn Cabeza: coloración general negra. Labro con leves puntuaciones, escotado en "v", con setas largas por los bordes. Setas cortas y esparcidas sobre la superficie. 
Clípeo subrugoso, con puntuación y setas cortas escasas sobre la superficie. Sutura clipeal no visible. Frente hundida, sin puntuación, con márgenes solevantado. Una arruga solevantada en el centro de la frente, la divide en dos, dándole un aspecto de dos hexágonos contiguos. Vertex levemente levantado. Mentón con leve rugosidad, con abundantes setas. Antenas de coloración negra con abundante pilosidad blanca, teniendo los últimos tres antenitos una apariencia aterciopelada. La extensión de las antenas llega hasta $3 / 4$ del pronoto. Tercer antenito más largo que el primero y segundo, antenitos cuarto al sexto de proporciones semejantes, antenitos séptimo y octavo más pequeños que los anteriores y de medida semejante, noveno y décimo antenito redondeados, antenito undécimo y final terminado en punta. Ojo compuesto, con presencia de setas por los bordes. Tórax: Pronoto plano, sin puntuación. Con setas esparcidas hacia los ángulos anteriores. alcanza su ancho pronotal máximo en centro de la estructura, donde forma un ángulo prominente, debido a la brusca estreches del la segunda mitad del pronoto. Este ángulo se proyecta a los lados en $90^{\circ}$, presentando una punta roma. Ángulo anterior redondeado hacia los extremos. Margen anterior con puntuación escasa, con abundante pilosidad corta que nace bajo este en dirección hacia la cabeza. Parte central del margen anterior levemente ensanchado, estrechándose hacia los costados. Margen lateral solevantado, grueso, glabro y liso. Angulo posterior proyectado hacia atrás, por sobre los élitros. Disco con dos carinas paralelas gruesas y muy levantadas, con presencia de escasa pero marcada rugosidad, corre casi longitudinal entre las carinas. Abundante arrugas entre las carinas y el margen lateral. Proepisterno liso y con pilosidad leve. Proesterno convexo, con puntuación aislada y presencia leve de setas. Apófisis proesternal subrectangular, con puntuación leve y setas esparcidas. Mesoepisterno con puntuación gruesa, esparcida y setas largas. Metaepisterno con pilosidad hacia los costados. Élitros: con presencia de setas diminutas y esparcida de color blanco en la mitad posterior de los élitros, entre las costas y entre las costas y el margen elitral, siendo un poco más densas en el declive elitral. Los élitros presentan una costa prominente en cada élitro, careciendo de costas secundarias. Sutura elitral solevantada. Espacios entre la sutura-costa lisa y espacio costa-margen con rugosidad levemente levantada (remanente de costa secundaria). Borde elitral notorio y crenulado. Epipleura y pseudopleura glabra y lisa. Abdomen: Esternitos glabros y de coloración general negra brillante y lisa, con dos depresiones, en el ventrito I y II. Ventrito V con finamente puntuado. Patas: Todas las patas de color negro. Coxas con pequeñas setas en la base y con puntuación leve. Trocánter con abundante


\section{$5,83 \mathrm{~mm}$}

FIgURA 1: Fotografía de paratipos en vista dorsal de Callyntra inconsiderata Zúñiga-Reinoso sp. nov. de la localidad de El Maitén: a) macho y b) hembra.

Figure 1: Paratypes photography in dorsal view of Callyntra inconsiderata Zúñiga-Reinoso sp. nov. from the locality El Maitén: a) male and b) female. 
pilosidad de coloración blanca. Fémures con presencia de setas de color blancas, más abundantes en su faz inferior, que en su faz superior. Tibias con pilosidad corta y gruesa que da apariencia de espinas por toda la superficie. Tarsos rodeados con abundantes setas. Cinco tarsos en las patas protoráxicas, los cuatro primero de dimensiones semejantes y quinto más largo, de tamaño 1:3. Cinco tarsos en las patas mesotoráxicas, primero más largo que los tarsitos segundo al cuarto, quinto tarso más largo que el primero. Cuatro tarsos en las patas metatoráxicas, primero y cuarto más largo que el segundo y tercero. Garras simples en todas las patas.

Genitalia: Edeago con los estilos del tegmen cóncavos, que se agudizan hacia el ápice. Margen basal bisinuado y mas ancho debido a unas leves proyecciones laterales, incompleto, formando un espacioamplio y semihexagonal en el ultimo tercio de los estilos. Con setas cortas y escasas en el segundo tercio de la superficie ventral, sobre el espacio semihexagonal. Láminas basales del tegmen con base redonda y espatulada. El lóbulo medio tubular, la mitad de ancho que los estilos laterales de tegmen, con la abertura apical alargada, ápice redondeado, y distalmente amplio (Fig. 2a). Al comparar la genitalia de C. inconsiderata, con las otras especies de Callyntra que son morfológicamente cercanas, se puede ver que esta estructura difiere con las otras especies (considerablemente con $C$. laticollis), debido al menor tamaño de la genitalia, a que posee los estilos del tegmen mas robustos, cortos y curvados y las láminas basales del tegmen más amplia (Fig 2b y c.). Además, $C$. paulseni presenta espacio de los estilos amplio y con forma lanceolada, no presenta proyecciones laterales en la base de los estilos. Mientra que $C$. laticollis presenta el espacio de los estilos estrecho, semicircular y estilos muchos mas largos que las otras dos especies .

VARIACIÓN INTRAESPECÍFICA.Se observa variación morfológica por localidad en los especímenes revisados. Se detallarán los caracteres de los individuos con distribución más septentrional, perteneciente a la localidad de Bucalemu. La localidad central irá en paréntesis redondos (i.e El Maitén, Navidad) y las del sur en paréntesis cuadrados [i.e Pupuya]. Presentan abundante pilosidad en los élitros (moderada) [muy escasa], pelos en la cara dorsal de las patas (moderada) [escasa o sin], carinas pronotales poco levantadas (levantadas) [levantadas] y costillas secundarias en los élitros notoria (moderadamente notoria) [prácticamente sin].
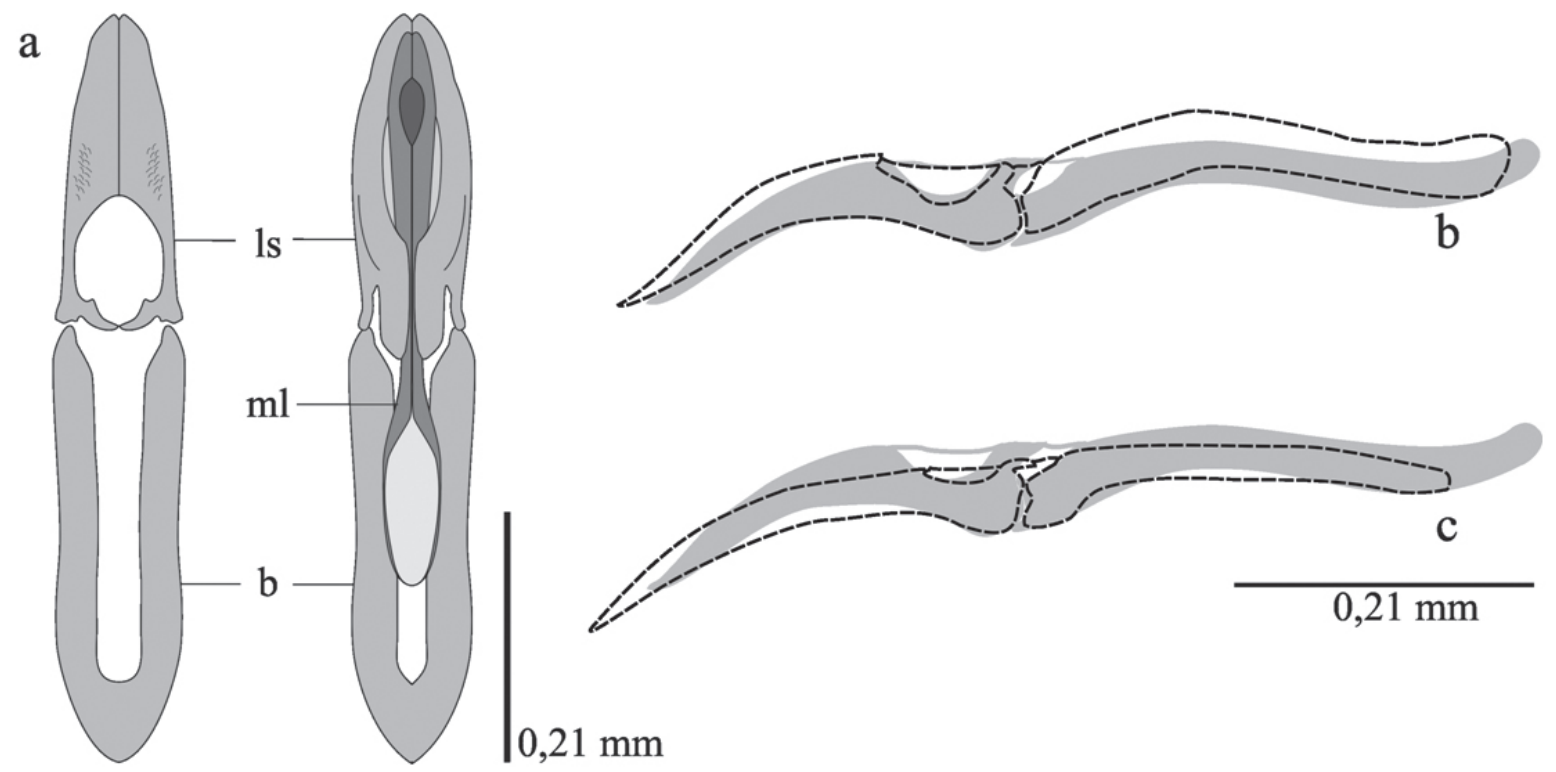

FiguRa 2. Genitalia macho de Callyntra inconsiderata Zúñiga-Reinoso y comparación de genitalia con especies morfológicamente más cercanas y con la especies donde fue considerada: a) Vista dorsal y ventral de la genitalia. b: lamina basal del tegmen; 1s: Estilos laterales del tegmen, ml: Lóbulo medio. b) Comparación en vista lateral de la genitalia de Callyntra inconsiderata (en gris), y de Callyntra paulseni (línea punteada negra). c) Comparación en vista lateral de la genitalia de Callyntra inconsiderata (en gris), y de Callyntra laticollis (línea punteada negra).

Figure 2. Male genitalia of Callyntra inconsiderata Zúñiga-Reinoso and genitalia comparison with specie morphologically close and the species which was considered: a) Dorsal and ventral views of the genitalia. b: basal lamina of tegmen; ls: lateral styles of tegmen, ml: median lobe. b) Comparison of lateral view of the aedeagus of Callyntra inconsiderata (in grey) and Callyntra paulseni (black dotted line). c) Comparison of lateral view of the aedeagus of Callyntra inconsiderata (in grey) and Callyntra laticollis (black dotted line). 
Dimorfismo SeXual. Se entregan entre paréntesis los caracteres de las hembras. Machos con marcada rugosidad longitudinal entre las carinas (rugosidad desordenada). Élitros sin costas secundarias (leves). Espacios elitral suturacosta lisa (arrugada). Ventritos lisos (arrugas verticales en los ventritos I, II y III). Machos con dos depresiones en el ventrito I y II (sin depresiones). Tarsos largos (tarsos cortos). El largo promedio de los machos es16.5 mm (D.E. \pm 1.16 ), mientras que el largo promedio de las hembras es de18.5 $\mathrm{mm}$ (D.E \pm 1.45$)$.

HÁBitAT. Todos los ejemplares han sido recolectados en quebradas con matorral y pastizales costeros. Caminan en las laderas de las quebradas, principalmente en aquellas con poca pendiente.

\section{DISCUSIÓN}

Esta revisión permitió establecer que el material que se encuentra en la costa de la $\mathrm{V}$ y VI región asignado bajo el nombre de $C$. laticollis, tantoen colecciones, como en publicaciones (eg Flores \& Vidal, 2000a), ZúñigaReinoso \& Cid, 2013), se trataría de una nueva especie que aquí nombramos como $C$. inconsiderata sp. nov. y que morfológicamente es cercana a la especie C. paulseni y en ningún caso cercana a $C$. laticollissensu stricto. En la descripción, se detallan tanto las característica morfológicas externas como del aedeagus, rasgos ampliamente utilizado para diferenciar las especies de Callyntra (ver Flores \& Vidal, 2000a; 2000b; Zúñiga-Reinoso \& Cid-Arcos, 2013; 2015; Aceituno \& Guerrero, 2014).

Por otra parte, en el material revisado se observa una variación morfológica por localidad en los especímenes de $C$. inconsiderada revisado, ya que los ejemplares con distribución mas septentrional (i.e Bucalemu), presentan mayor pilosidad en los élitros, tienen pelos en la cara dorsal de las patas, carinas pronotales menos levantadas y costillas secundarias en los élitros. La pilosidad en los élitros y patas y levantamiento de las carinas secundarias va disminuyendo hacia el sur, encontrándose ejemplares casi sin pilosidad en los élitros y patas y sin carinas secundarias en la localidad mas austral (i.e Pupuya). Por otra parte, se propone que el material proveniente de Bucalemu, debe ser estudiado con más detalle y mayor número de ejemplares, ya que sólo se contó con un solo ejemplar hembra e incompleto y que difiere bastante del material de localidades más australes. En general, las hembras fueron mas grandes que los machos, como lo documentado anteriormente por (Zúñiga-Reinoso \& Cid-Aros, 2013; 2015). Según Cloudsley-Thompson (2001), las diferencias en el tamaño entre sexo es una característica común, ya que es una adaptación de los tenebriónidos de ambientes áridos y semiáridos. Por otra parte, nuevamente se encontraron diferencias en algunas estructuras, como el tórax, abdomen, élitros y coxas (para mas detalles ver en descripción) entre sexos, como lo documentado para $C$. pehuenche por Zúñiga-Reinoso \& Cid-Aros (2015).

Finalmente, $C$. inconsiderata ha sido recolectada en pocas localidades costeras y se estima conveniente, que esta nueva especie endémica de las costas Chile central sea incluida en algún estado de conservación. Esto se debe a su acotado rango de distribución y al deterioro y fragmentación de su hábitat por el cambio del uso de suelo, dado el incremento significativo de plantaciones forestales en el sector. Estos parámetros han sido usados para determinar la categoría de amenaza de otras especies del género, como $C$. multicosta, $C$. cantillana, C. planiuscula y C. hibrida (ver Jerez et al. 2015).

\section{AGRADECIMIENTOS}

Los mas sinceros agradecimientos a Mario Elgueta, Patricia Estrada y Jorge Artigas, por permitir la revisión del material del Museo de Historia Natural, Colección Entomológica de la Universidad Metropolitana de Ciencias de la Educación y del Museo de Zoología de la Universidad de Concepción respectivamente. También agradecer a Mauricio Cid, Sebastian Larrea, Cesar Fortino y Pablo Pinto por la disposición de material para este estudio. Un especial agradecimiento a Guillermo Aceituno por las oportunas conversaciones previas a este manuscrito. A Dusan Boric, Carlos Muñoz, Jaime Zúñiga, Fermín Alfaro y Daniela Mardones por la compañía en las campañas de recolección. Finalmente, se agradece a las beca CONICYT Nro 21110367 y CONICYT Nro 8355/2014.

\section{BIBLIOGRAFÍA}

Aceituno, G. \& Guerrero, M.2014. Nuevas especies de Callyntra (Coleoptera: Tenebrionidae) de Chile central. Revista Chilena de Entomología39:05-11.

CloudsleyThompson, J.2001. Thermal and water relations of desert beetles. Naturwissenschaften 88:447460.

FLores, G.E. 1996. Estudio comparativo de las estructuras genitales en la tribu Nycteliini (Coleoptera: Tenebrionidae).Revista de la Sociedad Entomológica Argentina 55:3348.

Flores, G.E.2000. Cladistic analysis of the Neotropical tribe Nycteliini (Coleoptera: Tenebrionidae). Journal of the New York Entomological Society 108:13-25.

Flores, G. E. \& Vidal, P.2000a. Revalidation and systematic revision of the Chilean genus Callyntra Solier (Coleoptera: Tenebrionidae). Annals of the Entomological Society of America 93:10521075.

Flores, G.E. \& Vidal, P.2000b. Cladistic analysis of the Chilean genus Callyntra Solier (Coleoptera: Tenebrionidae), with description of a new species. Journal of the New York Entomological Society 108:187204. 
Jerez, V., Zúñiga-Reinoso, A., Muñoz-Escobar, C. \& PizarroAraYA, J.2015 Acciones y avances sobre la conservación de insectos en Chile. Gayana (Concepción) 79 (1):1-3.

PeÑA, L.E.1966. A preliminary attempt to divide Chile into entomofaunal regions, based on the Tenebrionidae (Coleoptera) Postilla 97:1-17.

Philippi, R. A. \& PhilipPI, F. H. 1864. Beschreibung eini- ger neuen Chilenischen Käfer. Stettiner Entomologische Zeitung 25:313-406.

Roig-Juñent, S., Flores, G.E., Ocampo,F.C. \& Smith,A.B. 2004. Nuevas citas de Coleoptera para la Argentina (Carabidae,
Lucanidae, Scarabaeidae y Tenebrionidae). Revista de la Sociedad Entomológica Argentina 63(3-4):45-48.

Vidal, P. \& Guerrero, M. 2007. Los Tenebriónidos de Chile. Ediciones Universidad Católica de Chile. Santiago, Chile $478 \mathrm{pp}$.

ZúñigaReinoso, A. \& CidArcos, M.2013.Callyntra hibrida n. sp.: Un nuevo tenebriónido de Chile central (Coleoptera: Tenebrionidae). Gayana (Concepción) 77(2):132135.

ZúñigaReinoso, A. \& CidArcos, M.2015. A new species of Callyntra (Coleoptera: Tenebrionidae) from central Chile. Zootaxa 4000 (2):294-298.

Recibido: 02.02.16

Aceptado: 09.09.16 